\title{
A general theorem on the stability of a class of functional equations including monomial equations
}

Yang-Hi Lee ${ }^{1}$ and Soon-Mo Jung ${ }^{2^{*}}$

\section{"Correspondence:}

smjung@hongik.ac.kr

${ }^{2}$ Mathematics Section, College of

Science and Technology, Hongik

University, Sejong, 339-701,

Republic of Korea

Full list of author information is

available at the end of the article

\begin{abstract}
In this paper, we prove a general theorem for the stability of a large class of $n$-dimensional functional equations of the form $D f\left(x_{1}, x_{2}, \ldots, x_{n}\right)=0$ by applying the direct method.
\end{abstract}

MSC: Primary 39B82; secondary 39B52

Keywords: generalized Hyers-Ulam stability; $n$-dimensional functional equation; monomial functional equation; direct method

\section{Introduction}

Let $V$ and $W$ be real vector spaces and let $n$ be a positive integer. For a given mapping $f: V \rightarrow W$, we define a mapping $D_{n} f: V \times V \rightarrow W$ by

$$
D_{n} f(x, y):=\sum_{i=0}^{n}(-1)^{n-i}{ }_{n} C_{i} f(i x+y)-n ! f(x)
$$

for all $x, y \in V$, where ${ }_{n} C_{i}=\frac{n !}{i !(n-i) !}$. A mapping $f: V \rightarrow W$ is called a monomial mapping of degree $n$ if $f$ satisfies the monomial functional equation $D_{n} f(x, y)=0$ for all $x, y \in V$. For example, a mapping $f: V \rightarrow W$ is called an additive mapping (or a quadratic mapping) if $f$ satisfies the functional equation $D_{1} f(x, y)=0\left(\right.$ or $\left.D_{2} f(x, y)=0\right)$ for all $x, y \in V$. We notice that if a mapping $f: V \rightarrow W$ is a monomial mapping of degree $n$, then $f(r x)=r^{n} f(x)$ for all $x \in V$ and all rational numbers $r$ (see [1,2]).

In the study of Hyers-Ulam stability problems of monomial functional equations, we have followed out a routine and monotonous procedure for proving the stability of the monomial functional equations under various conditions. We can find in the books [3-6] a lot of references concerning the Hyers-Ulam stability of functional equations (see also [7-12]).

In this paper, we prove a general stability theorem that can be easily applied to the (generalized) Hyers-Ulam stability of a large class of $n$-dimensional functional equations of the form $D f\left(x_{1}, x_{2}, \ldots, x_{n}\right)=0$, which includes the monomial functional equations.

(c) 2015 Lee and Jung. This article is distributed under the terms of the Creative Commons Attribution 4.0 International License (http://creativecommons.org/licenses/by/4.0/), which permits unrestricted use, distribution, and reproduction in any medium, provided you give appropriate credit to the original author(s) and the source, provide a link to the Creative Commons license, and indicate if changes were made. 
Throughout this paper, for a given mapping $f: V \rightarrow W$, we define a mapping $D f: V^{n} \rightarrow$ $W$ by

$$
D f\left(x_{1}, x_{2}, \ldots, x_{n}\right):=\sum_{i=1}^{m} c_{i} f\left(a_{i 1} x_{1}+a_{i 2} x_{2}+\cdots+a_{i n} x_{n}\right),
$$

where $m$ is a positive integer and $c_{i}, a_{i j}$ are real constants.

Indeed, this stability theorem can save us much trouble of proving the stability of relevant solutions repeatedly appearing in the stability problems for various functional equations (see [13-15]).

It should be remarked that Bahyrycz and Olko [16] applied the fixed point method to investigate the generalized Hyers-Ulam stability of the general linear equation

$$
\sum_{i=1}^{m} A_{i}\left(\sum_{i=1}^{n} a_{i j} x_{j}\right)+A=0 .
$$

Moreover, there are numerous recent results concerning the Hyers-Ulam stability of some particular cases of equation (1). Some of them were described in the survey paper [17].

In this paper, let $V$ be a real vector space and let $X$ and $Y$ be a real normed space resp. a real Banach space if there is no specification.

\section{Preliminaries}

We introduce a lemma from the paper [18], Corollary 3.5 .

Lemma 2.1 Let $k \neq 1$ be a positive real constant, let $b$ be a real constant, and let $Y$ be a real normed space. Assume, moreover, that $\phi: V \backslash\{0\} \rightarrow[0, \infty)$ is a function satisfying

$$
\Phi(x):=\sum_{i=0}^{\infty} \frac{\phi\left(k^{i} x\right)}{k^{b i}}<\infty
$$

for all $x \in V \backslash\{0\}$ and $f: V \rightarrow Y$ is an arbitrarily given mapping. If there exists a mapping $F: V \rightarrow Y$ satisfying

$$
\|f(x)-F(x)\| \leq \Phi(x)
$$

for all $x \in V \backslash\{0\}$ and

$$
F(k x)=k^{b} F(x)
$$

for all $x \in V$, then $F$ is a unique mapping satisfying (3) and (4).

We denote by $\mathbf{N}_{0}$ the set of all nonnegative integers, i.e., $\mathbf{N}_{0}=\mathbf{N} \cup\{0\}$.

Lemma 2.2 Let $k \neq 1$ be a positive real constant and let $b$ be a real constant. Assume that a function $\phi: V \backslash\{0\} \rightarrow[0, \infty)$ satisfies condition (2) for all $x \in V \backslash\{0\}$. If a mapping $f: V \rightarrow Y$ satisfies $f(0)=0$ and

$$
\left\|f(x)-\frac{f(k x)}{k^{b}}\right\| \leq \phi(x)
$$


for all $x \in V \backslash\{0\}$, then there exists a unique mapping $F: V \rightarrow Y$ satisfying (3) and (4) for all $x \in V \backslash\{0\}$ resp. for all $x \in V$.

Proof It follows from (5) that

$$
\begin{aligned}
\left\|\frac{f\left(k^{m} x\right)}{k^{b m}}-\frac{f\left(k^{m+l} x\right)}{k^{b(m+l)}}\right\| & \leq \sum_{i=m}^{m+l-1}\left\|\frac{f\left(k^{i} x\right)}{k^{b i}}-\frac{f\left(k^{i+1} x\right)}{k^{b(i+1)}}\right\| \\
& =\sum_{i=m}^{m+l-1} \frac{1}{k^{b i}}\left\|f\left(k^{i} x\right)-\frac{1}{k^{b}} f\left(k \cdot k^{i} x\right)\right\| \\
& \leq \sum_{i=m}^{m+l-1} \frac{\phi\left(k^{i} x\right)}{k^{b i}}
\end{aligned}
$$

for all $x \in V \backslash\{0\}$ and $m, l \in \mathbf{N}_{0}$. Thus, in view of (2), it is easy to show that the sequence $\left\{\frac{f\left(k^{m} x\right)}{k^{b m}}\right\}$ is a Cauchy sequence for all $x \in V \backslash\{0\}$. Since $Y$ is complete and $f(0)=0$, the sequence $\left\{\frac{f\left(k^{m} x\right)}{k^{b m}}\right\}$ converges for all $x \in V$. Hence, we can define a mapping $F: V \rightarrow Y$ by

$$
F(x):=\lim _{m \rightarrow \infty} \frac{f\left(k^{m} x\right)}{k^{b m}}
$$

for all $x \in V$.

We easily see that equality (4) is true by the definition of $F$. Moreover, we put $m=0$ and let $l \rightarrow \infty$ in (6) to get inequality (3). Obviously, in view of Lemma 2.1, the mapping $F: V \rightarrow Y$ satisfying (3) and (4) is uniquely determined.

Lemma 2.3 Let $k \neq 1, b, V, Y$ be as in Lemma 2.2, let $f: V \rightarrow Y$ and $F: V \rightarrow Y$ be arbitrary mappings, and let $n$ be a fixed integer greater than 1 . Assume that a function $\varphi:(V \backslash\{0\})^{n} \rightarrow$ $[0, \infty)$ satisfies the condition

$$
\sum_{i=0}^{\infty} \frac{\varphi\left(k^{i} x_{1}, k^{i} x_{2}, \ldots, k^{i} x_{n}\right)}{k^{b i}}<\infty
$$

for all $x_{1}, x_{2}, \ldots, x_{n} \in V \backslash\{0\}$. Iff satisfies the condition

$$
\left\|D f\left(x_{1}, x_{2}, \ldots, x_{n}\right)\right\| \leq \varphi\left(x_{1}, x_{2}, \ldots, x_{n}\right)
$$

for all $x_{1}, x_{2}, \ldots, x_{n} \in V \backslash\{0\}$ and if $F$ satisfies the equality

$$
D F\left(x_{1}, x_{2}, \ldots, x_{n}\right)=\lim _{m \rightarrow \infty} \frac{D f\left(k^{m} x_{1}, k^{m} x_{2}, \ldots, k^{m} x_{n}\right)}{k^{b m}}
$$

for all $x_{1}, x_{2}, \ldots, x_{n} \in V \backslash\{0\}$, then $F$ satisfies the equation

$$
D F\left(x_{1}, x_{2}, \ldots, x_{n}\right)=0
$$

for all $x_{1}, x_{2}, \ldots, x_{n} \in V \backslash\{0\}$. 
Proof It follows from (7), (8), and (9) that

$$
\begin{aligned}
\left\|D F\left(x_{1}, x_{2}, \ldots, x_{n}\right)\right\| & =\lim _{m \rightarrow \infty}\left\|\frac{D f\left(k^{m} x_{1}, k^{m} x_{2}, \ldots, k^{m} x_{n}\right)}{k^{b m}}\right\| \\
& \leq \lim _{m \rightarrow \infty} \frac{\varphi\left(k^{m} x_{1}, k^{m} x_{2}, \ldots, k^{m} x_{n}\right)}{k^{b m}} \\
& =0
\end{aligned}
$$

for all $x_{1}, x_{2}, \ldots, x_{n} \in V \backslash\{0\}$. In other words, $D F\left(x_{1}, x_{2}, \ldots, x_{n}\right)=0$ holds for all $x_{1}, x_{2}, \ldots, x_{n} \in$ $V \backslash\{0\}$.

Lemma 2.4 Let a be a real constant satisfying $a \notin\{-1,0,1\}$, let $N$ be an integer, and let $n$ be a fixed integer greater than 1 . Assume that $\mu: V \backslash\{0\} \rightarrow[0, \infty)$ is a function satisfying the condition

$$
\sum_{i=0}^{\infty} \frac{\mu\left(a^{i} x\right)}{|a|^{N i}}<\infty
$$

for all $x \in V \backslash\{0\}$. If a mapping $f: V \rightarrow Y$ satisfies $f(0)=0$ and

$$
\left\|f(a x)-a^{N} f(x)\right\| \leq \mu(x)
$$

for all $x \in V \backslash\{0\}$, then there exists a unique mapping $F: V \rightarrow Y$ satisfying

$$
F(a x)=a^{N} F(x)
$$

for all $x \in V$ and

$$
\|f(x)-F(x)\| \leq \sum_{i=0}^{\infty} \frac{\mu\left(a^{i} x\right)}{|a|^{N i+N}}
$$

for all $x \in V \backslash\{0\}$.

Proof It follows from (12) that

$$
\begin{aligned}
\left\|\frac{f\left(a^{m} x\right)}{a^{N m}}-\frac{f\left(a^{m+l} x\right)}{a^{N(m+l)}}\right\| & \leq \sum_{i=m}^{m+l-1}\left\|\frac{f\left(a^{i} x\right)}{a^{N i}}-\frac{f\left(a^{i+1} x\right)}{a^{N(i+1)}}\right\| \\
& =\sum_{i=m}^{m+l-1} \frac{\left\|f\left(a \cdot a^{i} x\right)-a^{N} f\left(a^{i} x\right)\right\|}{|a|^{N(i+1)}} \\
& \leq \sum_{i=m}^{m+l-1} \frac{\mu\left(a^{i} x\right)}{|a|^{N i+N}}
\end{aligned}
$$

for all $x \in V \backslash\{0\}$ and all $m, l \in \mathbf{N}_{0}$. Thus, in view of (11), it is easy to show that the sequence $\left\{\frac{f\left(a^{m} x\right)}{a^{N m}}\right\}$ is a Cauchy sequence for all $x \in V \backslash\{0\}$. Since $Y$ is complete and $f(0)=0$, 
the sequence $\left\{\frac{f\left(a^{m} x\right)}{a^{N m}}\right\}$ converges for all $x \in V$. Hence, we can define a mapping $F: V \rightarrow Y$ by

$$
F(x):=\lim _{m \rightarrow \infty} \frac{f\left(a^{m} x\right)}{a^{N m}}
$$

for all $x \in V$, from which it follows that (13).

Moreover, if we put $m=0$ and let $l \rightarrow \infty$ in (15), then we obtain inequality (14). Finally, using Lemma 2.1, we conclude that the mapping $F$ satisfying (13) and (14) is uniquely determined, since we have

$$
\|f(x)-F(x)\| \leq \sum_{i=0}^{\infty} \frac{\mu\left(a^{i} x\right)}{|a|^{N i+N}}=\sum_{i=0}^{\infty} \frac{|a|^{N} \mu\left(a^{2 i} x\right)+\mu\left(a \cdot a^{2 i} x\right)}{|a|^{2 N i+2 N}}=\sum_{i=0}^{\infty} \frac{\phi\left(k^{i} x\right)}{k^{N i}}
$$

for all $x \in V$, where we set $k:=a^{2}$ and $\phi(x):=\frac{\mu(x)}{|a|^{N}}+\frac{\mu(a x)}{|a|^{2 N}}$.

Lemma 2.5 Let a, N, $V, Y$ be defined as in Lemma 2.4, let $f: V \rightarrow Y$ and $F: V \rightarrow Y$ be arbitrary mappings, and let $n$ be a fixed integer greater than 1 . Assume that a function $\varphi:(V \backslash\{0\})^{n} \rightarrow[0, \infty)$ satisfies the condition

$$
\sum_{i=0}^{\infty} \frac{\varphi\left(a^{i} x_{1}, a^{i} x_{2}, \ldots, a^{i} x_{n}\right)}{|a|^{N i}}<\infty
$$

for all $x_{1}, x_{2}, \ldots, x_{n} \in V \backslash\{0\}$. Iff satisfies the inequality

$$
\left\|D f\left(x_{1}, x_{2}, \ldots, x_{n}\right)\right\| \leq \varphi\left(x_{1}, x_{2}, \ldots, x_{n}\right)
$$

for all $x_{1}, x_{2}, \ldots, x_{n} \in V \backslash\{0\}$, and if $F$ satisfies the condition

$$
D F\left(x_{1}, x_{2}, \ldots, x_{n}\right)=\lim _{m \rightarrow \infty} \frac{D f\left(a^{m} x_{1}, a^{m} x_{2}, \ldots, a^{m} x_{n}\right)}{a^{N m}}
$$

for all $x_{1}, x_{2}, \ldots, x_{n} \in V \backslash\{0\}$, then $F$ satisfies the equation

$$
D F\left(x_{1}, x_{2}, \ldots, x_{n}\right)=0
$$

for all $x_{1}, x_{2}, \ldots, x_{n} \in V \backslash\{0\}$.

Proof It easily follows from (16), (17), and (18) that

$$
\begin{aligned}
\left\|D F\left(x_{1}, x_{2}, \ldots, x_{n}\right)\right\| & =\lim _{m \rightarrow \infty}\left\|\frac{D f\left(a^{m} x_{1}, a^{m} x_{2}, \ldots, a^{m} x_{n}\right)}{a^{N m}}\right\| \\
& \leq \lim _{m \rightarrow \infty} \frac{\varphi\left(a^{m} x_{1}, a^{m} x_{2}, \ldots, a^{m} x_{n}\right)}{|a|^{N m}} \\
& =0
\end{aligned}
$$

for all $x_{1}, x_{2}, \ldots, x_{n} \in V \backslash\{0\}$, which implies that $D F\left(x_{1}, x_{2}, \ldots, x_{n}\right)=0$ holds for all $x_{1}, x_{2}$, $\ldots, x_{n} \in V \backslash\{0\}$. 


\section{Main results}

In this section, we denote by $V, X$, and $Y$ a real vector space, a real normed space, and a real Banach space, respectively, if there are no specifications.

We combine Lemmas 2.2 and 2.3 in the following theorem to formulate the main theorem which is easily applicable. We here notice that conditions (9) and (18) are satisfied in usual cases provided we apply the direct method for proving the (generalized) Hyers-Ulam stability of various functional equations of the form $D f\left(x_{1}, x_{2}, \ldots, x_{n}\right)=0$.

Theorem 3.1 Let $k \neq 1, b$, and $n>1$ be a positive real constant, a real constant, and an integer, respectively. Assume that $\phi: V \backslash\{0\} \rightarrow[0, \infty)$ is a function satisfying condition (2) for all $x \in V \backslash\{0\}$. If a mapping $f: V \rightarrow Y$ satisfies $f(0)=0$ and

$$
\left\|f(x)-\frac{f(k x)}{k^{b}}\right\| \leq \phi(x)
$$

for all $x \in V \backslash\{0\}$, then there exists a unique mapping $F: V \rightarrow Y$ satisfying

$$
\|f(x)-F(x)\| \leq \Phi(x)
$$

for all $x \in V \backslash\{0\}$ and

$$
F(k x)=k^{b} F(x)
$$

for all $x \in V$. In particular, if there exists a function $\varphi:(V \backslash\{0\})^{n} \rightarrow[0, \infty)$ satisfying condition (7) for all $x_{1}, x_{2}, \ldots, x_{n} \in V \backslash\{0\}$ and iff satisfies

$$
\left\|D f\left(x_{1}, x_{2}, \ldots, x_{n}\right)\right\| \leq \varphi\left(x_{1}, x_{2}, \ldots, x_{n}\right)
$$

and if $F$ satisfies

$$
D F\left(x_{1}, x_{2}, \ldots, x_{n}\right)=\lim _{m \rightarrow \infty} \frac{D f\left(k^{m} x_{1}, k^{m} x_{2}, \ldots, k^{m} x_{n}\right)}{k^{b m}}
$$

for all $x_{1}, x_{2}, \ldots, x_{n} \in V \backslash\{0\}$, then $F$ satisfies the equation

$$
D F\left(x_{1}, x_{2}, \ldots, x_{n}\right)=0
$$

for all $x_{1}, x_{2}, \ldots, x_{n} \in V \backslash\{0\}$.

In the following theorem, let $a$ be a real number not in $\{-1,0,1\}$. We can easily prove this theorem by using Lemmas 2.4 and 2.5. We also note that condition (18) is satisfied in usual cases when we use the direct method for proving the stability of functional equations of the form $D f\left(x_{1}, x_{2}, \ldots, x_{n}\right)=0$.

Theorem 3.2 Let $a \notin\{-1,0,1\}, N$, and $n>1$ be a real constant and integers, respectively. Assume that $\mu: V \backslash\{0\} \rightarrow[0, \infty)$ is a function satisfying condition (11) for all $x \in V \backslash\{0\}$. If a mapping $f: V \rightarrow Y$ satisfies $f(0)=0$ and

$$
\left\|f(a x)-a^{N} f(x)\right\| \leq \mu(x)
$$


for all $x \in V \backslash\{0\}$, then there exists a unique mapping $F: V \rightarrow Y$ such that

$$
F(a x)=a^{N} F(x)
$$

for all $x \in V$ and

$$
\|f(x)-F(x)\| \leq \sum_{i=0}^{\infty} \frac{\mu\left(a^{i} x\right)}{|a|^{N i+N}}
$$

for all $x \in V \backslash\{0\}$. In particular, if there exists a function $\varphi:(V \backslash\{0\})^{n} \rightarrow[0, \infty)$ satisfying condition (16) for all $x_{1}, x_{2}, \ldots, x_{n} \in V \backslash\{0\}$ and iff satisfies

$$
\left\|D f\left(x_{1}, x_{2}, \ldots, x_{n}\right)\right\| \leq \varphi\left(x_{1}, x_{2}, \ldots, x_{n}\right)
$$

and if $F$ satisfies

$$
D F\left(x_{1}, x_{2}, \ldots, x_{n}\right)=\lim _{m \rightarrow \infty} \frac{D f\left(a^{m} x_{1}, a^{m} x_{2}, \ldots, a^{m} x_{n}\right)}{a^{N m}}
$$

for all $x_{1}, x_{2}, \ldots, x_{n} \in V \backslash\{0\}$, then $F$ satisfies the equation

$$
D F\left(x_{1}, x_{2}, \ldots, x_{n}\right)=0
$$

for all $x_{1}, x_{2}, \ldots, x_{n} \in V \backslash\{0\}$.

We now introduce a corollary to Lemma 2.2.

Corollary 3.3 Assume that $k, b, p, \theta, \delta$ are real constants such that $k>0, k \neq 1, p \neq b$, $\theta>0$, and $\delta>0$. If a mapping $f: X \rightarrow Y$ satisfies $f(0)=0$ and

$$
\left\|f(k x)-k^{b} f(x)\right\| \leq \delta\|x\|^{p}
$$

for all $x \in X \backslash\{0\}$, then there exists a unique mapping $F: X \rightarrow Y$ satisfying (4) for all $x \in X$ and

$$
\|f(x)-F(x)\| \leq \frac{\delta\|x\|^{p}}{\left|k^{b}-k^{p}\right|}
$$

for all $x \in X \backslash\{0\}$.

Proof If $(k, p) \in(1, \infty) \times(-\infty, b)$ or $(k, p) \in(0,1) \times(b, \infty)$, then $\phi(x):=\frac{\delta}{k^{b}}\|x\|^{p}$ satisfies condition (2) with

$$
\Phi(x)=\frac{\delta\|x\|^{p}}{k^{b}-k^{p}} .
$$

For the case of $(k, p) \in(0,1) \times(-\infty, b)$ or $(k, p) \in(1, \infty) \times(b, \infty)$, we set $k^{\prime}:=\frac{1}{k}$ to get $\left(k^{\prime}, p\right) \in(1, \infty) \times(-\infty, b)$ or $\left(k^{\prime}, p\right) \in(0,1) \times(b, \infty)$, and

$$
\left\|f\left(k^{\prime} x\right)-k^{\prime b} f(x)\right\|=k^{\prime b}\left\|k^{b} f\left(\frac{x}{k}\right)-f(x)\right\| \leq k^{\prime b+p} \delta\|x\|^{p}
$$


for all $x \in X \backslash\{0\}$. By applying the same argument as in the first case, we can see that $\phi(x):=$ $k^{\prime p} \delta\|x\|^{p}$ satisfies condition (2) with

$$
\Phi(x)=\sum_{i=0}^{\infty} \frac{\phi\left(k^{\prime i} x\right)}{k^{\prime b i}}=\frac{\delta\|x\|^{p}}{k^{p}-k^{b}}
$$

for all $x \in X \backslash\{0\}$.

According to Lemma 2.2, there exists a unique mapping $F: X \rightarrow Y$ satisfying (4) for all $x \in X$ and (19) for all $x \in X \backslash\{0\}$.

The following corollary can be easily proved by using Lemma 2.4 .

Corollary 3.4 Assume that $N$ is an integer and $a, p, \theta, \delta$ are real constants such that $a \notin\{-1,0,1\}, p \neq N, \theta>0$, and $\delta>0$. If a mapping $f: X \rightarrow Y$ satisfies $f(0)=0$ and

$$
\left\|f(a x)-a^{N} f(x)\right\| \leq \delta\|x\|^{p}
$$

for all $x \in X \backslash\{0\}$, then there exists a unique mapping $F: X \rightarrow Y$ satisfying (13) for all $x \in X$ and

$$
\|f(x)-F(x)\| \leq \frac{\delta\|x\|^{p}}{\|\left. a\right|^{N}-|a|^{p} \mid}
$$

for all $x \in X \backslash\{0\}$.

Proof If $(|a|, p) \in(1, \infty) \times(-\infty, N)$ or $(|a|, p) \in(0,1) \times(N, \infty)$, then $\mu(x):=\delta\|x\|^{p}$ satisfies condition (11) with

$$
\sum_{i=0}^{\infty} \frac{\mu\left(a^{i} x\right)}{|a|^{N i}}=\frac{|a|^{N} \delta\|x\|^{p}}{|a|^{N}-|a|^{p}} .
$$

When $(|a|, p) \in(0,1) \times(-\infty, N)$ or $(|a|, p) \in(1, \infty) \times(N, \infty)$, we set $a^{\prime}:=\frac{1}{a}$ to get $\left(\left|a^{\prime}\right|, p\right) \in(1, \infty) \times(-\infty, N)$ or $\left(\left|a^{\prime}\right|, p\right) \in(0,1) \times(N, \infty)$, and

$$
\left\|f\left(a^{\prime} x\right)-a^{\prime N} f(x)\right\|=\left\|f\left(\frac{x}{a}\right)-\frac{1}{a^{N}} f(x)\right\| \leq \frac{1}{|a|^{N}} \delta\left\|\frac{x}{a}\right\|^{p}=\left|a^{\prime}\right|^{p+N} \delta\|x\|^{p}
$$

for all $x \in X \backslash\{0\}$. By arguing similarly as in the first case, we see that $\mu(x):=\left|a^{\prime}\right|^{p+N} \delta\|x\|^{p}$ satisfies condition (11) with

$$
\sum_{i=0}^{\infty} \frac{\mu\left(a^{\prime i} x\right)}{\left|a^{\prime}\right|^{N i}}=\frac{\left|a^{\prime}\right|^{p+2 N} \delta\|x\|^{p}}{\left|a^{\prime}\right|^{N}-\left|a^{\prime}\right|^{p}}=\frac{1}{|a|^{N}} \frac{\delta\|x\|^{p}}{|a|^{p}-|a|^{N}}
$$

for all $x \in X \backslash\{0\}$.

Therefore, by Lemma 2.4, there exists a unique mapping $F: X \rightarrow Y$ such that (13) and (21) hold for all $x \in X$ resp. for all $x \in X \backslash\{0\}$.

\section{Applications}

In this section, by $V, X$, and $Y$, we denote a real vector space, a real normed space, and a real Banach space, respectively, if there is no specification. 
For a given mapping $f: V \rightarrow Y$, we use the abbreviations

$$
\begin{aligned}
& D_{1} f(x, y):=f(a x+b y)-a f(x)-b f(y), \\
& D_{2} f(x, y):=f(a x+b y)+a b f(x-y)-a(a+b) f(x)-b(a+b) f(y),
\end{aligned}
$$

where $a$ and $b$ are fixed nonzero real numbers with $a+b \neq 0$ and $a b \neq 0$. (The definitions of $D_{1} f$ and $D_{2} f$ just above have nothing to do with the $D_{n} f$ given in the Introduction.)

Theorem 4.1 Let $a$ and $b$ be real constants such that $a+b \notin\{-1,0,1\}$ and let $\varphi$ : $(V \backslash\{0\})^{2} \rightarrow[0, \infty)$ be a function satisfying the condition

$$
\sum_{i=0}^{\infty} \frac{\varphi\left((a+b)^{i} x,(a+b)^{i} y\right)}{|a+b|^{i}}<\infty
$$

for all $x, y \in V \backslash\{0\}$. If a mapping $f: V \rightarrow Y$ satisfies $f(0)=0$ and

$$
\left\|D_{1} f(x, y)\right\| \leq \varphi(x, y)
$$

for all $x, y \in V \backslash\{0\}$, then there exists a unique mapping $F: V \rightarrow Y$ such that

$$
D_{1} F(x, y)=0
$$

for all $x, y \in V \backslash\{0\}$ and

$$
\|f(x)-F(x)\| \leq \sum_{i=0}^{\infty} \frac{\varphi\left((a+b)^{i} x,(a+b)^{i} x\right)}{|a+b|^{i+1}}
$$

for all $x \in V \backslash\{0\}$.

Proof It follows from (22) that

$$
\|(a+b) f(x)-f((a+b) x)\| \leq \varphi(x, x)
$$

for all $x \in V \backslash\{0\}$. If we set $a_{1}:=a+b, \mu(x):=\varphi(x, x), n:=2, N:=1$, and $D f:=D_{1} f$, then $a_{1}$, $N, f$, and $\mu$ satisfy conditions (11) and (12). According to Lemma 2.4 , there exists a unique mapping $F: V \rightarrow Y$ satisfying (13) and (14), i.e., $F$ satisfies

$$
F\left(a_{1} x\right)=a_{1}^{N} F(x)
$$

for all $x \in V$ and

$$
\|f(x)-F(x)\| \leq \sum_{i=0}^{\infty} \frac{\varphi\left(a_{1}^{i} x, a_{1}^{i} x\right)}{\left|a_{1}\right|^{N i+N}}
$$

for all $x \in V \backslash\{0\}$.

Moreover, $\varphi, f$, and $F$ satisfy (16) and (17). Condition (18) is also satisfied due to the definition of $F$ given in the proof of Lemma 2.4. Hence, in view of Theorem 3.2, the mapping $F$ satisfies (23) for all $x, y \in V \backslash\{0\}$. 
Theorem 4.2 Let $a$ and $b$ be real constants such that $a+b \notin\{-1,0,1\}$ and let $\varphi$ : $(V \backslash\{0\})^{2} \rightarrow[0, \infty)$ be a function satisfying the condition

$$
\sum_{i=0}^{\infty}|a+b|^{i} \varphi\left(\frac{x}{(a+b)^{i}}, \frac{y}{(a+b)^{i}}\right)<\infty
$$

for all $x, y, z \in V \backslash\{0\}$. If a mapping $f: V \rightarrow Y$ satisfies $f(0)=0$ and (22) for all $x, y \in V \backslash\{0\}$, then there exists a unique mapping $F: V \rightarrow Y$ satisfying (23) for all $x \in V$ and

$$
\|f(x)-F(x)\| \leq \sum_{i=0}^{\infty}|a+b|^{i} \varphi\left(\frac{x}{(a+b)^{i+1}}, \frac{x}{(a+b)^{i+1}}\right)
$$

for all $x \in V \backslash\{0\}$.

Proof It follows from (22) that

$$
\left\|\frac{1}{a+b} f(x)-f\left(\frac{x}{a+b}\right)\right\| \leq \frac{1}{|a+b|} \varphi\left(\frac{x}{a+b}, \frac{x}{a+b}\right)
$$

for all $x \in V \backslash\{0\}$. If we set $a_{1}:=\frac{1}{a+b}, \mu(x):=\left|a_{1}\right| \varphi\left(a_{1} x, a_{1} x\right), n:=2, N:=1$, and $D f:=D_{1} f$, then $a_{1}, N, f$, and $\mu$ satisfy conditions (11) and (12). In view of Lemma 2.4, there exists a unique mapping $F: V \rightarrow Y$ satisfying (13) and (14), i.e., $F$ satisfies

$$
F\left(a_{1} x\right)=a_{1}^{N} F(x)
$$

for all $x \in V$ and

$$
\|f(x)-F(x)\| \leq \sum_{i=0}^{\infty} \frac{\varphi\left(a_{1}^{i+1} x, a_{1}^{i+1} x\right)}{\left|a_{1}\right|^{N i+N-1}}
$$

for all $x \in V \backslash\{0\}$.

Furthermore, $\varphi, f$, and $F$ satisfy (16) and (17). The truth of (18) follows from the definition of $F$ given in the proof of Lemma 2.4. By Theorem 3.2, the mapping $F$ also satisfies (23) for all $x, y \in V \backslash\{0\}$.

Theorem 4.3 Let $a$ and $b$ be real constants satisfying $a+b \notin\{-1,0,1\}$ and let $\varphi$ : $(V \backslash\{0\})^{2} \rightarrow[0, \infty)$ be a function satisfying the condition

$$
\sum_{i=0}^{\infty} \frac{\varphi\left((a+b)^{i} x,(a+b)^{i} y\right)}{(a+b)^{2 i}}<\infty
$$

for all $x, y \in V \backslash\{0\}$. If a mapping $f: V \rightarrow Y$ satisfies $f(0)=0$ and

$$
\left\|D_{2} f(x, y)\right\| \leq \varphi(x, y)
$$

for all $x, y \in V \backslash\{0\}$, then there exists a unique mapping $F: V \rightarrow Y$ such that

$$
D_{2} F(x, y)=0
$$


for all $x, y \in V \backslash\{0\}$ and

$$
\|f(x)-F(x)\| \leq \sum_{i=0}^{\infty} \frac{\varphi\left((a+b)^{i} x,(a+b)^{i} x\right)}{(a+b)^{2 i+2}}
$$

for all $x \in V \backslash\{0\}$.

Proof It follows from (25) that

$$
\left\|(a+b)^{2} f(x)-f((a+b) x)\right\| \leq \varphi(x, x)
$$

for all $x \in V \backslash\{0\}$. If we set $a_{1}:=a+b, \mu(x):=\varphi(x, x), n:=2, N:=2$, and $D f:=D_{2} f$, then $a_{1}$, $N, f$, and $\mu$ satisfy conditions (11) and (12). We then see by Lemma 2.4 that there exists a unique mapping $F: V \rightarrow Y$ satisfying (13) and (14), i.e., $F$ satisfies

$$
F\left(a_{1} x\right)=a_{1}^{N} F(x)
$$

for all $x \in V$ and

$$
\|f(x)-F(x)\| \leq \sum_{i=0}^{\infty} \frac{\varphi\left(a_{1}^{i} x, a_{1}^{i} x\right)}{\left|a_{1}\right|^{N i+N}}
$$

for all $x \in V \backslash\{0\}$.

Furthermore, $\varphi, f$, and $F$ satisfy (16) and (17). Due to the definition of $F$ given in the proof of Lemma 2.4, we obtain the validity of (18). According to Theorem 3.2, the mapping $F$ satisfies (26) for all $x, y \in V \backslash\{0\}$.

Theorem 4.4 Let $a$ and $b$ be real constants such that $a+b \notin\{-1,0,1\}$ and let $\varphi$ : $(V \backslash\{0\})^{2} \rightarrow[0, \infty)$ be a function satisfying the condition

$$
\sum_{i=0}^{\infty}(a+b)^{2 i} \varphi\left(\frac{x}{(a+b)^{i}}, \frac{y}{(a+b)^{i}}\right)<\infty
$$

for all $x, y \in V \backslash\{0\}$. If a mapping $f: V \rightarrow Y$ satisfies $f(0)=0$ and (25) for all $x, y \in V \backslash\{0\}$, then there exists a unique mapping $F: V \rightarrow Y$ satisfying (26) for all $x, y \in V \backslash\{0\}$ and

$$
\|f(x)-F(x)\| \leq \sum_{i=0}^{\infty}(a+b)^{2 i} \varphi\left(\frac{x}{(a+b)^{i+1}}, \frac{x}{(a+b)^{i+1}}\right)
$$

for all $x \in V \backslash\{0\}$.

Proof It follows from (25) that

$$
\left\|\frac{1}{(a+b)^{2}} f(x)-f\left(\frac{x}{a+b}\right)\right\| \leq \frac{1}{(a+b)^{2}} \varphi\left(\frac{x}{a+b}, \frac{x}{a+b}\right)
$$

for all $x \in V \backslash\{0\}$. If we put $a_{1}:=\frac{1}{a+b}, \mu(x):=a_{1}^{2} \varphi\left(a_{1} x, a_{1} x\right), n:=2, N:=2$, and $D f:=D_{2} f$, then $a_{1}, N, f$, and $\mu$ satisfy conditions (11) and (12). It then follows from Lemma 2.4 that 
there exists a unique mapping $F: V \rightarrow Y$ satisfying (13) and (14), i.e., $F$ satisfies

$$
F\left(a_{1} x\right)=a_{1}^{N} F(x)
$$

for all $x \in V$ and

$$
\|f(x)-F(x)\| \leq \sum_{i=0}^{\infty} \frac{\varphi\left(a_{1}^{i+1} x, a_{1}^{i+1} x\right)}{\left|a_{1}\right|^{N i+N-2}}
$$

for all $x \in V \backslash\{0\}$.

Moreover, $\varphi, f$, and $F$ satisfy (16) and (17). From the definition of $F$ given in the proof of Lemma 2.4, it follows that (18) is true. In view of Theorem 3.2, the mapping $F$ satisfies (26) for all $x, y \in V \backslash\{0\}$.

\section{Competing interests}

The authors declare that they have no competing interests.

\section{Authors' contributions}

All authors contributed equally to the writing of this paper. All authors read and approved the final manuscript.

\section{Author details}

'Department of Mathematics Education, Gongju National University of Education, Gongju, 314-711, Republic of Korea.

${ }^{2}$ Mathematics Section, College of Science and Technology, Hongik University, Sejong, 339-701, Republic of Korea.

\section{Acknowledgements}

The authors are grateful to anonymous referees for their kind suggestions and comments. Soon-Mo Jung was supported by Basic Science Research Program through the National Research Foundation of Korea (NRF) funded by the Ministry of Education (No. 2013R1A1A2005557).

Received: 14 April 2015 Accepted: 23 August 2015 Published online: 04 September 2015

\section{References}

1. Baker, JA: A general functional equation and its stability. Proc. Am. Math. Soc. 133, 1657-1664 (2005)

2. Lee, Y-H: On the stability of the monomial functional equation. Bull. Korean Math. Soc. 45, 397-403 (2008)

3. Cho, Y-J, Rassias, TM, Saadati, R: Stability of Functional Equations in Random Normed Spaces. Springer Optimization and Its Applications, vol. 86. Springer, New York (2013)

4. Czerwik, S: Functional Equations and Inequalities in Several Variables. World Scientific, Hackensack (2002)

5. Hyers, DH, Isac, G, Rassias, TM: Stability of Functional Equations in Several Variables. Birkhäuser, Boston (1998)

6. Jung, S-M: Hyers-Ulam-Rassias Stability of Functional Equations in Nonlinear Analysis. Springer Optimization and Its Applications, vol. 48. Springer, New York (2011)

7. Brillouët-Belluot, N, Brzdęk, J, Ciepliński, K: On some recent developments in Ulam's type stability. Abstr. Appl. Anal. 2012, Article ID 716936 (2012)

8. Găvruţa, P: A generalization of the Hyers-Ulam-Rassias stability of approximately additive mappings. J. Math. Anal. Appl. 184, 431-436 (1994)

9. Hyers, DH: On the stability of the linear functional equation. Proc. Natl. Acad. Sci. USA 27, $222-224$ (1941)

10. Lee, Y-H, Jung, S-M: A general uniqueness theorem concerning the stability of additive and quadratic functional equations. J. Funct. Spaces 2015, Article ID 643969 (2015)

11. Rassias, TM: On the stability of the linear mapping in Banach spaces. Proc. Am. Math. Soc. 72, 297-300 (1978)

12. Ulam, SM: A Collection of Mathematical Problems. Interscience, New York (1960)

13. Almahelabi, M, Sirouni, M, Charifi, A, Kabboj, S: Fuzzy stability of the monomial functional equation. J. Nonlinear Anal. Appl. 2014, Article ID jnaa-00244 (2014)

14. Eskandani, GZ, Găvruţa, P, Kim, G-H: On the stability problem in fuzzy Banach space. Abstr. Appl. Anal. 2012, Article ID 763728 (2012)

15. Zaharia, C: On the probabilistic stability of the monomial functional equation. J. Nonlinear Sci. Appl. 6, 51-59 (2013)

16. Bahyrycz, A, Olko, J: On stability of the general linear equation. Aequ. Math. (2015). doi:10.1007/s00010-014-0317-z

17. Brzdęk, J, Ciepliński, K: Hyperstability and superstability. Abstr. Appl. Anal. 2013, Article ID 401756 (2013)

18. Lee, Y-H, Jung, S-M: A general uniqueness theorem concerning the stability of monomial functional equations in fuzzy spaces. J. Inequal. Appl. 2015, 66 (2015) 